J. Korean Math. Soc. 50 (2013), No. 3, pp. 627-639

http://dx.doi.org/10.4134/JKMS.2013.50.3.627

\title{
SEMILINEAR NONLOCAL DIFFERENTIAL EQUATIONS WITH DELAY TERMS
}

\author{
Jin-Mun JeOng And Su Jin CheOn
}

\begin{abstract}
The goal of this paper is to obtain the regularity and the existence of solutions of a retarded semilinear differential equation with nonlocal condition by applying Schauder's fixed point theorem. We construct the fundamental solution, establish the Hölder continuity results concerning the fundamental solution of its corresponding retarded linear equation, and prove the uniqueness of solutions of the given equation.
\end{abstract}

\section{Introduction}

In this paper, we deal with the nonlocal initial value problem governed by retarded semilinear parabolic type equations in a Hilbert space as follows.

$$
\left\{\begin{array}{l}
\frac{d}{d t} x(t)=A_{0} x(t)+\int_{-h}^{0} a(s) A_{1} x(t+s) d s \\
\quad+f\left(t, x(t), x\left(b_{1}(t)\right), \ldots, x\left(b_{m}(t)\right)\right)+k(t), t \geq 0, \\
x(0)=g^{0}-\phi(x), \quad x(s)=g^{1}(s)-e^{s} \phi(x), \quad-h \leq s<0,
\end{array}\right.
$$

Let $H$ and $V$ be complex Hilbert spaces such that the embedding $V \subset H$ is continuous. Let $A_{0}$ be the operator associated with a bounded sesquilinear form defined in $V \times V$ satisfying Gårding inequality. Then $A_{0}$ generates an analytic semigroup $S(t)$ in both $H$ and $V^{*}$ and so the equation (NRE) may be considered as an equation in both $H$ and $V^{*}$. The operator $A_{1}$ is bounded linear from $V$ to $V^{*}$ such that its restriction to $D\left(A_{0}\right)$ is a bounded linear operator from $D\left(A_{0}\right)$ to $H$. The function $a(\cdot)$ is assumed to be real-valued and Hölder continuous in the interval $[-h, 0]$, and $f, \phi, b_{i}(i=1, \ldots, m)$ are given functions satisfying some assumptions.

Received September 26, 2012.

2010 Mathematics Subject Classification. Primary 35F25, 35K55.

Key words and phrases. asemilinear differential equation, nonlocal condition, delay term, fundamental solution, regularity for solutions.

This research was supported by Basic Science Research Program through the National research Foundation of Korea(NRF) funded by the Ministry of Education, Science and Technology(2012-0007560). 
In view of the maximal regularity result by Di Blasio, Kunisch and Sinestrari [1], the retarded functional differential equation of parabolic type

$$
\left\{\begin{array}{l}
\frac{d}{d t} x(t)=A_{0} x(t)+\int_{-h}^{0} a(s) A_{1} x(t+s) d s+k(t), \\
x(0)=g^{0}, \quad x(s)=g^{1}(s), \quad-h \leq s<0
\end{array}\right.
$$

has a unique solution $x$ in the class $L^{2}\left(0, T ; D\left(A_{0}\right)\right) \cap W^{1,2}(0, T ; H)$ (or see $[2,3]$ for the case of class $\left.L^{2}(0, T ; V) \cap W^{1,2}\left(0, T ; V^{*}\right)\right)$. There are many papers on the existence of solutions of nonlocal abstract initial value problems without delay (see the bibliographies in $[4,5]$ ). Results on the existence of mild and classical solutions of nonlocal Cauchy problem for a semilinear functional differential evolution equation was obtained by Byszewski and Akca [5].

In recent years, Obukhovski and Zecca [6] discussed the controllability for the system governed by semilinear differential inclusions in a Banach space with noncompact semigroup and Xue [7, 8] studied semilinear nonlocal problems without the assumption of compactness in Banach spaces. Zhu et el. [9] concerned the impulsive differential equations with nonlocal conditions in general Banach spaces.

In this paper, we extend these results to the equation (NRE) with unbounded principal operators and delay term. Let $W(\cdot)$ be the fundamental solution of the linear equation associated with (RE), which is defined to be the operator valued function satisfying

$$
\begin{aligned}
\frac{d}{d t} W(t) & =A_{0} W(t)+\int_{-h}^{0} a(s) A_{1} W(t+s) d s \\
W(0) & =I, \quad W(s)=0, \quad s \in[-h, 0) .
\end{aligned}
$$

The fundamental solution enables us to solve the equation (NRE). For the basis of our arguments, we construct the fundamental solution in the sense of Nakagiri [10] to (RE), establish the Hölder continuity results concerning the fundamental solution $W(t)$ of the equation (RE) and obtain the regularity and the existence of solutions of (NRE) by applying Schauder's fixed point theorem. According to Tanabe [11, Theorem 1], we will also prove the uniqueness of solutions of the equation (NRE).

\section{Semilinear equation and its fundamental solution}

The inner product and the norm in $H$ are denoted by $(\cdot, \cdot)$ and $|\cdot|$, respectively. $V$ is another Hilbert space densely and continuously embedded in $H$. The notations $\|\cdot\|$ and $\|\cdot\|_{*}$ denote the norms of $V$ and of $V^{*}$ as usual, respectively. For brevity, we may regard that

$$
\|u\|_{*} \leq|u| \leq\|u\|, \quad u \in V .
$$

Let $B(\cdot, \cdot)$ be a bounded sesquilinear form defined in $V \times V$ and satisfying Gårding's inequality

$$
\operatorname{Re} B(u, u) \geq c_{0}\|u\|^{2}-c_{1}|u|^{2}, \quad c_{0}>0, \quad c_{1} \geq 0 .
$$


Let $A_{0}$ be the operator associated with the sesquilinear form $-B(\cdot, \cdot)$ :

$$
\left(A_{0} u, v\right)=-B(u, v), \quad u, v \in V .
$$

It follows from (2.2) that for every $u \in V$,

$$
\operatorname{Re}\left(\left(c_{1}-A_{0}\right) u, u\right) \geq c_{0}\|u\|^{2} .
$$

Then $A_{0}$ is a bounded linear operator from $V$ to $V^{*}$, and its realization in $H$ which is the restriction of $A_{0}$ to

$$
D\left(A_{0}\right)=\left\{u \in V ; A_{0} u \in H\right\}
$$

is also denoted by $A_{0}$. Then $A_{0}$ generates an analytic semigroup $S(t)=e^{t A_{0}}$ in both $H$ and $V^{*}$ as in Theorem 3.6.1 of [12]. Hence we may assume that $0 \in \rho\left(A_{0}\right)$ according to the Lax-Milgram theorem, where $\rho\left(A_{0}\right)$ denotes the resolvent set of $A_{0}$. Moreover, there exists a constant $C_{0}$ such that

$$
\|u\| \leq C_{0}\|u\|_{D\left(A_{0}\right)}^{1 / 2}|u|^{1 / 2},
$$

for every $u \in D\left(A_{0}\right)$, where

$$
\|u\|_{D\left(A_{0}\right)}=\left(\left|A_{0} u\right|^{2}+|u|^{2}\right)^{1 / 2}
$$

is the graph norm of $D\left(A_{0}\right)$.

For the sake of simplicity, we assume that $S(t)$ is uniformly bounded. Then

$$
|S(t)| \leq M_{0},\left|A_{0} S(t)\right| \leq M_{0} / t,\left|A_{0}^{2} S(t)\right| \leq M_{0} / t^{2}, t>0
$$

for some constant $M_{0}$ (e.g., [8]). We also assume that $a(\cdot)$ is Hölder continuous of order $\rho$ :

$$
|a(\cdot)| \leq H_{0}, \quad|a(s)-a(\tau)| \leq H_{1}(s-\tau)^{\rho}
$$

for some constants $H_{0}, H_{1}$.

Lemma 2.1. For $0<s<t$ and $0<\alpha<1$,

$$
\begin{aligned}
& |S(t)-S(s)| \leq \frac{M_{0}}{\alpha}\left(\frac{t-s}{s}\right)^{\alpha}, \\
& \left|A_{0} S(t)-A_{0} S(s)\right| \leq M_{0}(t-s)^{\alpha} s^{-\alpha-1} .
\end{aligned}
$$

Proof. From (2.4), for $0<s<t$,

$$
|S(t)-S(s)|=\left|\int_{s}^{t} A_{0} S(\tau) d \tau\right| \leq M_{0} \log \frac{t}{s} .
$$

It is easily seen that for any $t>0$ and $0<\alpha<1$,

$$
\begin{aligned}
\log (1+t) & =\int_{1}^{1+t} \frac{1}{s} d s \leq \int_{1}^{1+t} \frac{1}{s^{1-\alpha}} d s \\
& =\frac{1}{\alpha}\left\{(1+t)^{\alpha}-1\right\} \leq t^{\alpha} / \alpha .
\end{aligned}
$$


Combining (2.9) with (2.8), we get (2.6). For $0<s<t$,

$$
\left|A_{0} S(t)-A_{0} S(s)\right|=\left|\int_{s}^{t} A_{0}^{2} S(\tau) d \tau\right| \leq M_{0}(t-s) / t s .
$$

Noting that $(t-s) / t \leq((t-s) / s)^{\alpha}$ for $0<\alpha<1$, we obtain (2.7) from $(2.10)$.

First, we introduce the following linear retarded functional differential equation:

$$
\frac{d}{d t} x(t)=A_{0} x(t)+\int_{-h}^{0} a(s) A_{1} x(t+s) d s+k(t) .
$$

Let $W(\cdot)$ be the fundamental solution of the above linear equation in the sense of Nakagiri [10], which is the operator valued function satisfying

$$
\left\{\begin{array}{l}
\frac{d}{d t} W(t)=A_{0} W(t)+\int_{-h}^{0} a(s) A_{1} W(t+s) d s, \\
W(0)=I, \quad W(s)=0 \quad s \in[-h, 0) .
\end{array}\right.
$$

According to Duhamel's principle, the problem mentioned above is transformed to the following integral equation:

$$
\left\{\begin{array}{l}
W(t)=S(t)+\int_{0}^{t} S(t-s) \int_{-h}^{0} a(\tau) A_{1} W(s+\tau) d \tau d s, \quad t>0 \\
W(0)=I, \quad W(s)=0 \quad-h \leq s<0
\end{array}\right.
$$

where $S(\cdot)$ is the semigroup generated by $A_{0}$. Then

$$
\left\{\begin{aligned}
x(t) & =W(t)\left(g^{0}-\phi(x)\right)+\int_{-h}^{0} U_{t}(s)\left(g^{1}(s)-e^{s} \phi(x)\right) d s \\
& +\int_{0}^{t} W(t-s)\left\{f\left(s, x(s), x\left(b_{1}(s)\right), \ldots, x\left(b_{m}(s)\right)\right)+k(s)\right\} d s \\
U_{t}(s) & =\int_{-h}^{s} W(t-s+\sigma) a(\sigma) A_{1} d \sigma
\end{aligned}\right.
$$

Recalling the formulation of mild solutions, we know that the mild solution of $(\mathrm{RE})$ is also represented by

$$
x(t)=\left\{\begin{array}{l}
S(t)\left(g^{0}-\phi(x)\right)+\int_{0}^{t} S(t-s)\left\{\int_{-h}^{0} a(\tau) A_{1} x(s+\tau) d \tau\right. \\
\left.+f\left(s, x(s), x\left(b_{1}(s)\right), \ldots, x\left(b_{m}(s)\right)\right)+k(s)\right\} d s, 0 \leq t \\
g^{1}(s)-e^{s} \phi(x), \quad-h \leq s<0 .
\end{array}\right.
$$

According to H. Tanabe [11], we set

$$
V(t)=\left\{\begin{array}{l}
A_{0}(W(t)-S(t)), \quad t \in(0, h] \\
A_{0} W(t), \quad t \in(n h,(n+1) h], n=1,2, \ldots
\end{array}\right.
$$

For $0<t \leq h$,

$$
W(t)=S(t)+A_{0}^{-1} V(t)
$$

and from (2.11), we have

$$
W(t)=S(t)+\int_{0}^{t} \int_{\tau}^{t} S(t-s) a(\tau-s) d s A_{1} W(\tau) d \tau .
$$


Hence

$$
V(t)=V_{0}(t)+\int_{0}^{t} A_{0} \int_{\tau}^{t} S(t-s) a(\tau-s) d s A_{1} A_{0}^{-1} V(\tau) d \tau
$$

where

$$
V_{0}(t)=\int_{0}^{t} A_{0} \int_{\tau}^{t} S(t-s) a(\tau-s) d s A_{1} S(\tau) d \tau .
$$

For $n h \leq t \leq(n+1) h(n=1,2, \ldots)$, the fundamental solution $W(t)$ is represented by

$$
\begin{aligned}
W(t)= & S(t)+\int_{0}^{t-h} \int_{\tau}^{\tau+h} S(t-s) a(\tau-s) d s A_{1} W(\tau) d \tau \\
& +\int_{t-h}^{n h} \int_{\tau}^{t} S(t-s) a(\tau-s) d s A_{1} W(\tau) d \tau \\
& +\int_{n h}^{t} \int_{\tau}^{t} S(t-s) a(\tau-s) d s A_{1} W(\tau) d \tau
\end{aligned}
$$

The integral equation to be satisfied by $(2.13)$ is

$$
V(t)=V_{0}(t)+\int_{n h}^{t} A_{0} \int_{\tau}^{t} S(t-s) a(\tau-s) d s A_{1} A_{0}^{-1} V(\tau) d \tau
$$

where

$$
\begin{aligned}
V_{0}(t)= & A_{0} S(t)+\int_{0}^{t-h} A_{0} \int_{\tau}^{\tau+h} S(t-s) a(\tau-s) d s A_{1} W(\tau) d \tau \\
& +\int_{t-h}^{n h} A_{0} \int_{0}^{t} S(t-s) a(\tau-s) d s A_{1} W(\tau) d \tau .
\end{aligned}
$$

Thus the integral equation (2.13) can be solved by successive approximations and $V(t)$ is uniformly bounded in $[n h,(n+1) h]$ :

$$
\sup _{n h \leq t \leq(n+1) h}|V(t)|<\infty, \quad n=0,1,2, \ldots
$$

It is not difficult to show that for $n>1$,

$V_{0}(n h+0) \neq V_{0}(n h-0), W(n h+0)=W(n h-0)$ and $V(n h+0)=V(n h-0)$.

Lemma 2.2. There exists a constant $C_{n}^{\prime}>0$ such that

$$
\left|\int_{n h}^{t} a(\tau-s) A_{1} W(\tau) d \tau\right| \leq C_{n}^{\prime}
$$

for $n=0,1,2, \ldots, t \in[n h,(n+1) h]$ and $t \leq s \leq t+h$.

Proof. For $t \in(0, h]$ (i.e., $n=0)$, from (2.13) it follows

$$
\begin{aligned}
& \int_{0}^{t} a(\tau-s) A_{1} W(\tau) d \tau=\int_{0}^{t} a(\tau-s) A_{1} A_{0}^{-1}\left(A_{0} S(\tau)+V(\tau)\right) d \tau \\
= & \int_{0}^{t}(a(\tau-s)-a(s)) A_{1} A_{0}^{-1} A_{0} S(\tau) d \tau+a(s) A_{1} A_{0}^{-1}(S(t)-I)
\end{aligned}
$$




$$
+\int_{0}^{t} a(\tau-s) A_{1} A_{0}^{-1} V(\tau) d \tau
$$

Noting that

$$
\left|\int_{0}^{t}(a(\tau-s)-a(s)) A_{1} A_{0}^{-1} A_{0} S(\tau) d \tau\right| \leq M_{0} H_{1}\left|A_{1} A_{0}^{-1}\right| \int_{0}^{t} \tau^{\rho-1} d \tau,
$$

we have

$$
\begin{aligned}
\left|\int_{0}^{t} a(\tau-s) A_{1} W(\tau) d \tau\right| \leq & \left|A_{1} A_{0}^{-1}\right|\left\{\rho^{-1} h^{\rho} M_{0} H_{1}+H_{0}\left(M_{0}+1\right)\right. \\
& \left.+h H_{0}\left(\sup _{0 \leq t \leq h}|V(t)|\right)\right\} .
\end{aligned}
$$

Thus the assertion (2.14) holds in $[0, h]$. For $t \in[n h,(n+1) h], n \geq 1$,

$$
\int_{n h}^{t} a(\tau-s) A_{1} W(\tau) d \tau=\int_{n h}^{t} a(\tau-s) A_{1} A_{0}^{-1} V(\tau) d \tau .
$$

The right-hand side of the above equality is estimated as

$$
\left|\int_{n h}^{t} a(\tau-s) A_{1} A_{0}^{-1} V(\tau) d \tau\right| \leq h H_{0}\left|A_{1} A_{0}^{-1}\right|\left(\sup _{n h \leq t \leq(n+1) h}|V(t)|\right) .
$$

Hence we get the assertion (2.14).

Proposition 2.3. The fundamental solution $W(t)$ of $(R E)$ exists uniquely. For $0<t<t^{\prime} \leq n h, n>1$, there exists a constant $C_{n}>0$ such that

$$
\left|W\left(t^{\prime}\right)-W(t)\right| \leq C_{n}\left(t^{\prime}-t\right)^{\alpha}, \quad 0<\alpha<1 .
$$

Proof. The existence and uniqueness of the fundamental solution $W(t)$ of (RE) is due to Tanabe [11]. With the aid of suitable changes in variables, from (2.11) we obtain

$$
W(t)=\left\{\begin{array}{l}
S(t)+\int_{0}^{t} S(t-s) \int_{0}^{s} a(\tau-s) A_{1} W(\tau) d \tau d s, 0<t \leq h, \\
S(t)+\int_{0}^{t} S(t-s) \int_{s-h}^{s} a(\tau-s) A_{1} W(\tau) d \tau d s, h<t .
\end{array}\right.
$$

For $0<t \leq h$, since

$$
\begin{aligned}
\left|W\left(t^{\prime}\right)-W(t)\right| \leq & \left|S\left(t^{\prime}\right)-S(t)\right| \\
& +\left|\int_{0}^{t}\left(S\left(t^{\prime}-s\right)-S(t-s)\right) \int_{0}^{s} a(\tau-s) A_{1} W(\tau) d \tau d s\right| \\
& +\left|\int_{t}^{t^{\prime}} S\left(t^{\prime}-s\right) \int_{0}^{s} a(\tau-s) A_{1} W(\tau) d \tau d s\right|,
\end{aligned}
$$

from Lemmas 2.1 and 2.2, it follows that

$$
\left|W\left(t^{\prime}\right)-W(t)\right| \leq \text { const. }\left(\frac{t^{\prime}-t}{t}\right)^{\alpha} \leq C_{n}\left(t^{\prime}-t\right)^{\alpha}, \quad 0<\alpha<1 .
$$

For $h<t$, we get (2.15) analogously. 
Considering as an equation in $V^{*}$, we also obtain the same norm estimates of $(2.4)-(2.7),(2.15)$ in the space $V^{*}$. By virtue of Theorem 3.3 of [2], [4] we have the following regularity results on the retarded linear equation $(\mathrm{RE})$.

Proposition 2.4. 1) Let $F:=\left(D\left(A_{0}\right), H\right)_{\frac{1}{2}, 2}$, where $\left(D\left(A_{0}\right), H\right)_{1 / 2,2}$ denotes the real interpolation space between $D\left(A_{0}\right)$ and $H$. For $\left(g^{0}, g^{1}\right) \in F \times L^{2}(-h, 0$; $\left.D\left(A_{0}\right)\right)$ and $k \in L^{2}(0, T ; H), T>0$, there exists a unique solution $x$ of $(R E)$ belonging to

$$
L^{2}\left(-h, T ; D\left(A_{0}\right)\right) \cap W^{1,2}(0, T ; H) \subset C([0, T] ; F)
$$

and satisfying

$$
\begin{aligned}
& \|x\|_{L^{2}\left(-h, T ; D\left(A_{0}\right)\right) \cap W^{1,2}(0, T ; H)} \\
\leq & C_{T}\left(\left\|g^{0}\right\|_{F}+\left\|g^{1}\right\|_{L^{2}\left(-h, 0 ; D\left(A_{0}\right)\right)}+\|k\|_{L^{2}(0, T ; H)}\right),
\end{aligned}
$$

where $C_{T}$ is a constant depending on $T$.

2) Let $\left(g^{0}, g^{1}\right) \in H \times L^{2}(-h, 0 ; V)$ and $k \in L^{2}\left(0, T ; V^{*}\right), T>0$. Then there exists a unique solution $x$ of $(R E)$ belonging to

$$
L^{2}(-h, T ; V) \cap W^{1,2}\left(0, T ; V^{*}\right) \subset C([0, T] ; H)
$$

and satisfying

$$
\|x\|_{L^{2}(-h, T ; V) \cap W^{1,2}\left(0, T ; V^{*}\right)} \leq C_{T}\left(\left|g^{0}\right|+\left\|g^{1}\right\|_{L^{2}(-h, 0 ; V)}+\|k\|_{L^{2}\left(0, T ; V^{*}\right)}\right) .
$$

\section{Existence and uniqueness of solutions}

In this section, we investigate the regularity for solutions of the equation (NRE) with the operator $A_{0}$ associated with the sesquilinear form $-B(\cdot, \cdot)$ satisfying Gårding's inequality

$$
\operatorname{Re} B(u, u) \geq c_{0}\|u\|^{2}, \quad c_{0}>0 .
$$

Hence we have $0 \in \rho\left(A_{0}\right)$. In what follows, we assume that the embedding $D\left(A_{0}\right) \hookrightarrow V$ is compact. Then $A_{0}^{-1}: H \rightarrow D\left(A_{0}\right) \hookrightarrow V \hookrightarrow H$ is compact. This is equivalent to saying that the semigroup $S(t)$ is completely continuous $[13$, Corollary 3.4], and hence $W(t)$ defined as in (2.11) is completely continuous (for more information on the fundamental solution, see [14, Proposition 3.1] or [15, Lemma 2.4]). For brevity, we assume that

$$
\|W(t)\| \leq M_{1}, \quad t>0
$$

Let $T>0$ be fixed and $X=C([0, T] ; H)$. Put

$$
H_{r}=\{z \in H:|z| \leq r\} \text { and } X_{r}=\left\{x \in X:\|x\|_{X} \leq r\right\}
$$

for some $r>0$.

Let $k \in L^{2}(0, T ; H)$ and let $f:[0, T] \times H^{m+1} \rightarrow H, \phi: X \rightarrow H, b_{i}:[0, T] \rightarrow$ $[0, T](i=1, \ldots, m)$ satisfying the following assumptions: 
Assumption (A). (i) $f \in C\left([0, T] \times H^{m+1} ; H\right), \phi \in C(X ; H)$ and $b_{i} \in$ $C\left([0, T] ; \mathbb{R}^{+}\right)(i=1, \ldots, m)$. Moreover, there are $L_{i}>0(i=1,2)$ such that

$$
\left|f\left(s, z_{0}, z_{1}, \ldots, z_{m}\right)\right| \leq L_{1} \text { for } s \in[0, T], z_{i} \in H_{r}(i=1, \ldots, m) .
$$

(ii) $\phi$ is completely continuous such that

$$
|\phi(x)| \leq L_{2} \text { for } x \in X_{r} .
$$

Lemma 3.1. Let $h \in L^{2}(0, T ; H)$. Then for any $t>0$, the operators $P_{t}$ and $Q_{t}$ from $L^{2}(0, T ; H)$ into $H$ defined by

$$
P_{t} h=\int_{0}^{t} S(t-s) h(s) d s \quad \text { and } \quad Q_{t} h=\int_{0}^{t} W(t-s) h(s) d s
$$

are completely continuous.

Proof. We define the $\epsilon$-approximation $P_{t}^{\epsilon}: L^{2}(0, T ; H) \rightarrow H$ of $P_{t}$ for $\epsilon \in(0, t]$ by

$$
P_{t}^{\epsilon} h=S(\epsilon) \int_{0}^{t-\epsilon} S(t-\epsilon-s) h(s) d s .
$$

Since $S(t)$ is completely continuous, so is $P_{t}^{\epsilon}$. The complete continuity of $P_{t}$ follows from

$$
\left|\left(P_{t}^{\epsilon}-P_{t}\right) h\right| \leq\left.\sqrt{\epsilon} M|| h\right|_{L^{2}(0, T ; H)} .
$$

The $\epsilon$-approximation $Q_{t}^{\epsilon}: L^{2}(0, T ; H) \rightarrow H$ of $Q_{t}$ is defined by

$$
Q_{t}^{\epsilon} h=\int_{0}^{t-\epsilon} W(t-s) h(s) d s .
$$

Noting that

$W\left(t+t^{\prime}\right)=S\left(t^{\prime}\right) W(t)+\int_{0}^{t^{\prime}} S\left(t^{\prime}-\sigma\right) \int_{-h}^{0} a(\tau) A_{1} x(\sigma+t+\tau) d \tau d \sigma, \quad 0<t, t^{\prime} \leq T$,

we have

$$
\begin{aligned}
Q_{t}^{\epsilon} h= & S(\epsilon) \int_{0}^{t-\epsilon} W(t-\epsilon-s) h(s) d s \\
& +\int_{0}^{t-\epsilon} \int_{0}^{\epsilon} S(\epsilon-\sigma) \int_{0}^{-h^{\prime}} a(\tau) A_{1} x(\sigma+t-\epsilon-s+\tau) d \tau d \sigma d s .
\end{aligned}
$$

By using a similar procedure to the case of $P_{t}$, we obtain that $Q_{t}$ is completely continuous from the complete continuity of $W(t)$ and $Q_{t}^{\epsilon}$.

Theorem 3.2. Let $\left(g^{0}, g^{1}\right) \in H \times L^{2}\left(-h, 0 ; D\left(A_{0}\right)\right)$ and $k \in L^{2}(0, T ; H)$. Assume that $f, \phi$ and $b_{i}(i=1, \ldots, m)$ satisfy Asuumption $(A)$. Then there exists a mild solution $x$ of $(N R E)$ belonging to $C([0, T] ; H)$. Furthermore, if $g^{0}-\phi(x) \in F=\left(D\left(A_{0}\right), H\right)_{\frac{1}{2}, 2}$, then the solution $x$ of $(N R E)$ belongs to

$$
L^{2}\left(-h, T ; D\left(A_{0}\right)\right) \cap W^{1,2}(0, T ; H) \subset C([0, T] ; F)
$$


SEMILINEAR NONLOCAL DIFFERENTIAL EQUATIONS WITH DELAY TERMS 635 and satisfies

$$
\begin{aligned}
& \|x\|_{L^{2}\left(-h, T ; D\left(A_{0}\right)\right) \cap W^{1,2}(0, T ; H)} \\
\leq & C_{T}^{\prime}\left(1+\left\|g^{0}\right\|_{\left(D\left(A_{0}\right), H\right)_{\frac{1}{2}, 2}}+\left\|g^{1}\right\|_{L^{2}\left(-h, 0 ; D\left(A_{0}\right)\right)}+\|k\|_{L^{2}(0, T ; H)}\right),
\end{aligned}
$$

where $C_{T}^{\prime}$ is a constant depending on $T$.

Proof. Let

$$
\begin{aligned}
r= & M_{1}\left(\left|g^{0}\right|+L_{2}\right)+h M_{1} H_{0}\left\|A_{1} A_{0}^{-1}\right\|\left(L_{2} h+\sqrt{h}\left\|g^{1}\right\|_{L^{2}\left(-h, 0: D\left(A_{0}\right)\right)}\right) \\
& +M_{1} L_{1} T+M_{1} \sqrt{T}\|k\|_{L^{2}(0, T ; H)} .
\end{aligned}
$$

Define a mapping $\mathcal{F}$ on $X_{r}$ by the fomular

$$
\begin{aligned}
(\mathcal{F} x)(t)= & W(t)\left(g^{0}-\phi(x)\right)+\int_{-h}^{0} U_{t}(s)\left(g^{1}(s)-e^{s} \phi(x)\right) d s \\
& +\int_{0}^{t} W(t-s)\left\{f\left(s, x(s), x\left(b_{1}(s)\right), \ldots, x\left(b_{m}(s)\right)\right)+k(s)\right\} d s .
\end{aligned}
$$

In view of (3.1) and Assumption (A),

$$
\begin{aligned}
|(\mathcal{F} x)(t)| \leq & M_{1}\left(\left|g^{0}\right|+L_{2}\right)+h M_{1} H_{0}\left\|A_{1} A_{0}^{-1}\right\|\left(L_{2} h+\sqrt{h}\left\|g^{1}\right\|_{L^{2}\left(-h, 0: D\left(A_{0}\right)\right)}\right) \\
& +M_{1} L_{1} T+M_{1} \sqrt{T}\|k\|_{L^{2}(0, T ; H)},
\end{aligned}
$$

then $\mathcal{F}\left(X_{r}\right) \subset X_{r} \subset C([0, T] ; H)$. Observe that $0<t<t^{\prime} \leq T$. From (3.1), Assumption (A) and Proposition 2.3, we have

$$
\begin{aligned}
& \left|x\left(t^{\prime}\right)-x(t)\right| \\
\leq & \left|\left(W\left(t^{\prime}\right)-W(t)\right)\left(g^{0}-\phi(x)\right)\right| \\
& +\int_{-h}^{0} \int_{-h}^{s}\left|\left(W\left(t^{\prime}-s+\sigma\right)-W(t-s+\sigma)\right) a(\sigma) A_{1}\left(g^{1}(s)-e^{s} \phi(x)\right)\right| d \sigma d s \\
& +\int_{0}^{t}\left|W\left(t^{\prime}-s\right)-W(t-s)\right|\left|f\left(s, x(s), x\left(b_{1}(s)\right), \ldots, x\left(b_{m}(s)\right)\right)+k(s)\right| d s \\
& +\int_{t}^{t^{\prime}}\left|W\left(t^{\prime}-s\right)\right|\left|f\left(s, x(s), x\left(b_{1}(s)\right), \ldots, x\left(b_{m}(s)\right)\right)+k(s)\right| d s \\
\leq & C_{n}\left(t^{\prime}-t\right)^{\alpha}\left(\left|g^{0}-\phi(x)\right|+T L_{1}+\sqrt{T}\|k\|_{L^{2}(0, T ; H)}\right) \\
& \left.+C_{n} \int_{-h}^{0} \int_{-h}^{s}\left(t^{\prime}-t\right)^{\alpha} H_{0}\left\|A_{1} A_{0}^{-1}\right\|_{B(H, H)} \| g^{1}(s)-e^{s} \phi(x)\right) \|_{D\left(A_{0}\right)} d \sigma d s \\
& +M_{1} L_{1}\left(t^{\prime}-t\right)+\int_{t}^{t^{\prime}}|k(s)| d s \\
\leq & C_{n}\left(t^{\prime}-t\right)^{\alpha}\left(\left|g^{0}-\phi(x)\right|+T L_{1}+\sqrt{T}\|k\|_{L^{2}(0, T ; H)}\right)
\end{aligned}
$$




$$
\begin{aligned}
& \left.+C_{n} H_{0}\left\|A_{1} A_{0}^{-1}\right\|_{B(H, H)}\left(t^{\prime}-t\right)^{\alpha} \int_{-h}^{0} \| g^{1}(s)-e^{s} \phi(x)\right) \|_{D\left(A_{0}\right)} d s \\
& +M_{1} L_{1}\left(t^{\prime}-t\right)+\left(t^{\prime}-t\right)^{1 / 2}\|k\|_{L^{2}(0, T ; H)} .
\end{aligned}
$$

Thus if $0<\kappa \leq \frac{1}{2}$, then we have

$$
\left|x\left(t^{\prime}\right)-x(t)\right| \leq \text { const. }\left(t^{\prime}-t\right)^{\kappa}\left(1+\left|g^{0}\right|+\left\|g^{1}\right\|_{L^{2}\left(-h, 0 ; D\left(A_{0}\right)\right)}+\|k\|_{L^{2}(0, T ; H)}\right) .
$$

Hence $\mathcal{F}\left(X_{r}\right)$ is a uniformly equicontinuous family of functions. Furthermore, from (2.17) in Proposition 2.4 and Assumption (A) it follows that

$$
\begin{aligned}
|(\mathcal{F} x)(t)| \leq & \|\mathcal{F} x\|_{C([0, T] ; H)} \\
\leq & C_{T}\left(\left|g^{0}-\phi(x)\right|+\left\|g^{1}-e \cdot \phi(x)\right\|_{L^{2}(-h, 0 ; V)}\right. \\
& \| f\left(\cdot, x(\cdot), x\left(b_{1}(\cdot)\right), \ldots, x\left(b_{m}(\cdot)\right)+k \|_{L^{2}\left(0, T ; V^{*}\right)}\right) \\
\leq & \text { const. }\left(1+\left|g^{0}\right|+\left\|g^{1}\right\|_{L^{2}\left(-h, 0 ; D\left(A_{0}\right)\right)}+\|k\|_{L^{2}(0, T ; H)}\right) .
\end{aligned}
$$

Thus $\mathcal{F}\left(X_{r}\right)$ is equibounded.

From Lemma 3.1 it follows that the set $V(t)=\left\{(\mathcal{F} x)(t): x \in X_{r}\right\}$ is relatively compact in $H$. By (ii) of Assumption (A), $V(0)$ is obviously is relatively compact. The proof for the continuity of $\mathcal{F}$ is routine, and thus is omitted. Therefore, applying Schauder's fixed point theorem, it holds that $\mathcal{F}$ has a fixed point in $X_{r}$, and hence any fixed point of $\mathcal{F}$ is a mild solution of (NRE).

Assume that $g^{0}-\phi(x) \in F=\left(D\left(A_{0}\right), H\right)_{\frac{1}{2}, 2}$. Then in virtue of Proposition 2.4 , there exists a solution $x$ of (NRE) belonging to

$$
L^{2}\left(-h, T ; D\left(A_{0}\right)\right) \cap W^{1,2}(0, T ; H) \subset C([0, T] ; F)
$$

and satisfying

$$
\begin{aligned}
& \|x\|_{L^{2}\left(-h, T ; D\left(A_{0}\right)\right) \cap W^{1,2}(0, T ; H)} \\
\leq & C_{1}^{\prime}\left(\left\|g^{0}-\phi(x)\right\|_{\left(D\left(A_{0}\right), H\right)_{\frac{1}{2}, 2}}+\left\|g^{1}-e \cdot \phi(x)\right\|_{L^{2}\left(-h, 0 ; D\left(A_{0}\right)\right)}+\|k\|_{L^{2}(0, T ; H)}\right) \\
\leq & C_{2}^{\prime}\left(1+\left\|g^{0}\right\|_{\left(D\left(A_{0}\right), H\right)_{\frac{1}{2}, 2}}+\left\|g^{1}\right\|_{L^{2}\left(-h, 0 ; D\left(A_{0}\right)\right)}+\|k\|_{L^{2}(0, T ; H)}\right) .
\end{aligned}
$$

Theorem 3.3. Suppose that the functions $f, \phi$ and $b_{i}(i=1, \ldots, m)$ satisfy Assumption $(A), g^{1}$ is a Hölder continuous function in $[-h, 0]$ with values in $D\left(A_{0}\right)$, and $k$ is a Hölder continuous function in $[0, T]$ with values in $H$. Assume, additionally, that

(i) there exists a constant $L_{3}>0$ such that

$$
\begin{aligned}
& \left|f\left(s, z_{0}, z_{1}, \ldots, z_{m}\right)-f\left(\tilde{s}, \tilde{z}_{0}, \tilde{z}_{1}, \ldots, \tilde{z}_{m}\right)\right| \leq L_{3}\left(|s-\tilde{s}|+\sum_{i=0}^{m}\left\|z_{i}-\tilde{z}_{i}\right\|\right) \\
& \text { for } s, \tilde{s} \in I, z_{i}, \tilde{z}_{i} \in H_{r}(i=0,1, \ldots, m),
\end{aligned}
$$

where $r$ is the constant in (3.2), 
SEMILINEAR NONLOCAL DIFFERENTIAL EQUATIONS WITH DELAY TERMS 637

(ii) $x$ is a solution of problem $(N R E)$ and there is a constant $\mathcal{H}>0$ such that

$$
\left|x\left(b_{i}(s)\right)-x\left(b_{i}(\tilde{s})\right)\right| \leq \mathcal{H}|x(s)-x(\tilde{s})| \quad \text { for } s, \tilde{s} \in I .
$$

Then $x$ represented as in $(2.12)$ is the unique solution of (NRE) satisfying the initial condition

$$
x(s)=g^{1}(s)-e^{s} \phi(x), \quad s \in[-h, 0] .
$$

Proof. Put

$$
\begin{aligned}
& G(s)=g^{1}(s)-e^{s} \phi(s), s \in[-h, 0] \\
& K(t)=f\left(t, x(t), x\left(b_{1}(t)\right), \ldots, x\left(b_{m}(t)\right)\right)+k(t), t \in[0, T] .
\end{aligned}
$$

Then in virtue of Theorem 2 of [11], it is sufficient to prove that $G$ and $K$ are Lipschitz continuous in $[-h, 0]$ and in $[0, T]$, respectively. Since $g^{1}$ is a Hölder continuous function in $[-h, 0]$ with values in $D\left(A_{0}\right)$ and

$$
\begin{aligned}
\left|e^{s^{\prime}} \phi(x)-e^{s} \phi(x)\right| & =\left|\int_{0}^{1} \frac{d}{d \sigma}\left(e^{s^{\prime} \sigma} e^{s(1-\sigma)}\right) \phi(x) d \sigma\right| \\
& \leq \int_{0}^{1}\left|e^{s^{\prime} \sigma} e^{s(1-\sigma)}\left(s^{\prime}-s\right) \phi(x)\right| d \sigma \\
& \leq\left(s^{\prime}-s\right) e^{s^{\prime}}\|\phi(x)\|_{D\left(A_{0}\right)},
\end{aligned}
$$

it holds that $G$ is Hölder continuous. Furthermore, since

$$
\begin{aligned}
& \left|K\left(t^{\prime}\right)-K(t)\right| \\
\leq & \left|k\left(t^{\prime}\right)-k(t)\right| \\
& +\left|f\left(t^{\prime}, x\left(t^{\prime}\right), x\left(b_{1}\left(t^{\prime}\right)\right), \ldots, x\left(b_{m}\left(t^{\prime}\right)\right)\right)-f\left(t, x(t), x\left(b_{1}(t)\right), \ldots, x\left(b_{m}(t)\right)\right)\right| \\
\leq & \left|k\left(t^{\prime}\right)-k(t)\right|+L_{3}\left(\left|t^{\prime}-t\right|+\sum_{i=1}^{m}\left|x\left(b_{i}\left(t^{\prime}\right)\right)-x\left(b_{i}(t)\right)\right|\right) \\
\leq & \left|k\left(t^{\prime}\right)-k(t)\right|+L_{3}\left(\left|t^{\prime}-t\right|+m \mathcal{H}\left|x\left(t^{\prime}\right)-x(t)\right|\right),
\end{aligned}
$$

from (3.3) and the Hölder continuity of $k$, it follows that $K$ is Hölder continuous in $[0, T]$.

\section{Example}

Let

$$
\begin{aligned}
& H=L^{2}(0, \pi), V=H_{0}^{1}(0, \pi), V^{*}=H^{-1}(0, \pi), \\
& B(u, v)=\int_{0}^{\pi} \frac{d u(x)}{d x} \frac{\frac{d v(x)}{d x}}{d x}
\end{aligned}
$$

and

$$
A_{i}=d^{2} / d x^{2}(i=0,1) \quad \text { with } \quad D\left(A_{i}\right)=\left\{y \in H^{2}(0, \pi): y(0)=y(\pi)=0\right\} .
$$


We consider the following nonlinear term:

$$
f\left(s, z_{0}, z_{1}, \ldots, z_{m}\right)=l(s)+\frac{\gamma \sum_{i=1}^{m} z_{i}}{1+\left|\sum_{i=1}^{m} z_{i}\right|}, \quad \gamma \in \mathbb{R},
$$

where

$$
|l(s)-l(\tilde{s})| \leq \sigma|s-\tilde{s}|, \quad l(0)=0,
$$

which comes out in a feedback control system for a diffusion and reaction process in a enzyme membrane. Then

$$
\begin{gathered}
\left|f\left(s, z_{0}, z_{1}, \ldots, z_{m}\right)\right| \leq \sup _{0 \leq t \leq T}|l(t)|+|\gamma|, \\
\quad\left|f\left(s, z_{0}, z_{1}, \ldots, z_{m}\right)-f\left(\tilde{s}, \tilde{z}_{0}, \tilde{z}_{1}, \ldots, \tilde{z}_{m}\right)\right| \\
\leq|l(s)-l(\tilde{s})|+\frac{|\gamma|\left(1+2\left|\sum_{i=1}^{m} \tilde{z}_{i}\right|\right)\left(\sum_{i=1}^{m} z_{i}-\sum_{i=1}^{m} \tilde{z}_{i}\right)}{\left(1+\left|\sum_{i=1}^{m} z_{i}\right|\right)\left(1+\left|\sum_{i=1}^{m} \tilde{z}_{i}\right|\right)} \\
\leq \sigma|s-\tilde{s}|+2|\gamma| \sum_{i=1}^{m}\left|z_{i}-\tilde{z}_{i}\right| .
\end{gathered}
$$

Let $t_{1}, \ldots, t_{p}$ be given real numbers such that $0<t_{1}<\ldots<t_{p}<T$. Then we can give $\phi$ by the formula

$$
\phi(x)=\sum_{i=1}^{p} d_{i} x\left(t_{i}\right) \quad x \in C\left([0, T] ; L^{2}(0, \pi)\right),
$$

where $d_{i}(i=1, \ldots, p)$ are given constants. Let the solution $x$ be represented by the following retarded semilinear parabolic type equation:

$$
\left\{\begin{array}{l}
\frac{d}{d t} x(t)=A_{0} x(t)+\int_{-h}^{0} a(s) A_{1} x(t+s) d s \\
\quad+f\left(t, x(t), x\left(b_{1}(t)\right), \ldots, x\left(b_{m}(t)\right)\right)+k(t), t \geq 0, \\
x(0)=g^{0}-\sum_{i=1}^{p} d_{i} x\left(t_{i}\right), \quad x(s)=g^{1}(s)-e^{s} \phi(x), \quad-h \leq s<0,
\end{array}\right.
$$

where the forcing term $k$ belongs to $L^{2}\left(0, T ; V^{*}\right), b_{i}(t)=t(i=1, \ldots, m)$. Then the nonlinear term $f, \phi$ and $b_{i}(i=1, \ldots, m)$ satisfy the conditions of Theorems 3.2 and 3.3 .

\section{References}

[1] G. Di Blasio, K. Kunisch and E. Sinestrari, $L^{2}$-regularity for parabolic partial integro differential equations with delay in the highest-order derivatives, J. Math. Anal. Appl. 102 (1984), no. $1,38-57$.

[2] J. M. Jeong, Stabilizability of retarded functional differential equation in Hilbert space, Osaka J. Math. 28 (1991), no. 2, 347-365.

[3] _ Retarded functional differential equations with $L^{1}$-valued controller, Funkcial. Ekvac. 36 (1993), no. 1, 71-93.

[4] K. Balachandran, Existence of solutions of a delay differential equation with nonlocal condition, Indian J. Pure Appl. Math. 27 (1996), no. 5, 443-449.

[5] L. Byszewski and H. Akca, Existence of solutions of a semilinear functional-differential evolution nonlocal problem, Nonlinear Anal. 34 (1998), no. 1, 65-72. 
[6] V. Obukhovski and P. Zecca, Controllability for systems governed by semilinear differential inclusions in a Banach space with a noncompact semigroup, Nonlinear Anal. 70 (2009), no. 9, 3424-3436.

[7] X. M. Xue, Nonlocal nonlinear differential equations with a measure of noncompactness in Banach spaces, Nonlinear Anal. 70 (2009), no. 7, 2593-2601.

[8] - Semilinear nonlocal problems without the assumptions of compactness in Banach spaces, Anal. Appl. 8 (2010), no. 2, 211-225.

[9] L. Zhu, Q. Dong and G. Li, Impulsive differential equations with nonlocal condition in general Banach spaces, Adv. Difference Equ. 2012 (2012), no. 10, 1-21.

[10] S. Nakagiri, Optimal control of linear retarded systems in Banach spaces, J. Math. Anal. Appl. 120 (1986), no. 1, 169-210.

[11] H. Tanabe, Fundamental solutions for linear retarded functional differential equations in Banach space, Funkcial. Ekvac. 35 (1992), no. 1, 149-177.

[12] H. Tanabe, Equations of Evolution, Pitman-London, 1979.

[13] A. Pazy, Semigroups of Linear Operators and Applications to Partial Differential Equations, Springer-Verlag New York, 1983.

[14] S. Nakagiri, Structural properties of functional differential equations in Banach spaces, Osaka J. Math. 25 (1988), no. 2, 353-398.

[15] L. Wang, Approximate controllability for integrodifferential equations and multiple delays, J. Optim. Theory Appl. 143 (2009), no. 1, 185-206.

Jin-Mun JEONG

Department of Applied Mathematics

Pukyong NATional University

Busan 608-737, Korea

E-mail address: jmjeong@pknu.ac.kr

SU JiN CHEON

Department of Mathematics

Pukyong National University

BUSAN 609-737, KoreA

E-mail address: hjwh12@hanmail.net 